\title{
Detection of relic gravitational waves in thermal case by using Adv.LIGO data of GW150914
}

\author{
Basem Ghayour $^{1, a}$, Jafar Khodagholizadeh ${ }^{2, b}$ \\ ${ }^{1}$ School of Physics, University of Hyderabad, Hyderabad 500 046, India \\ ${ }^{2}$ Farhangian University, P. O. Box 11876-13311, Tehran, Iran
}

Received: 15 July 2017 / Accepted: 8 August 2017 / Published online: 18 August 2017

(C) The Author(s) 2017. This article is an open access publication

\begin{abstract}
The thermal spectrum of relic gravitational waves enhances the usual spectrum. Our analysis shows that there exist some chances for detection of the thermal spectrum in addition to the usual spectrum by comparison with sensitivity of Adv.LIGO of GW150914 and detector based on the maser light. The behavior of the inflation and reheating stages are often known as power law expansion like $S(\eta) \propto \eta^{1+\beta}, S(\eta) \propto \eta^{1+\beta_{s}}$, respectively, with constraints $1+\beta<0,1+\beta_{s}>0$. The $\beta$ and $\beta_{s}$ have an unique effect on the shape of the spectrum. We find some values of the $\beta$ and $\beta_{s}$ by considering the mentioned comparison. As obtained results give us more information about the evolution of inflation and reheating stages.
\end{abstract}

\section{Introduction}

The relic gravitational waves (RGWs) have a wide range of frequency $\sim\left(10^{-19}-10^{11} \mathrm{~Hz}\right)$. They are generated before and during the inflation stage. They did not have interaction with other matter during their travel from the early universe until now. Therefore they contain valuable information as regards the early universe. Thus we can obtain the information by detecting RGWs on the whole range of the frequency. Also nascent GW astronomy will permit one to ultimately discuss the consistence of Einstein's general relativity or of other, alternative gravitational theories, as has been clarified in [1]. Nowadays we are trying to detect the waves at different frequency ranges, like $\sim\left(10^{-19}-10^{-16} \mathrm{~Hz}\right)$ by Planck [2], $\sim\left(10^{-7}-10^{0} \mathrm{~Hz}\right)$ by eLISA [3], $\sim\left(10^{-1}-10^{4} \mathrm{~Hz}\right)$ by Advanced.LIGO (Adv.LIGO) [4], $\sim\left(10^{0}-10^{4} \mathrm{~Hz}\right)$ by Einstein telescope (ET) [5], GHz band by detector based on the maser light (Dml) [6] etc. If the inflation were preceded by a radiation era, then there would be thermal spectrum of grav-

\footnotetext{
a e-mail: ba.ghayour@gmail.com

b e-mail: gholizadeh@ipm.ir
}

itational waves at the time of inflation [7-9]. It is believed that the thermal spectrum of RGWs may affect the CMB temperature and the polarization anisotropies in the low frequency range $\sim\left(10^{-18}-10^{-16} \mathrm{~Hz}\right)[7-9]$. Also, in the high frequency range $\sim\left(10^{9}-10^{11} \mathrm{~Hz}\right)$, extra dimensions [10-15] cause a thermal spectrum of gravitational waves (or, equivalently, a primordial background of gravitons) [16]. According to the extra-dimensional models, the thermal gravitons with a very high frequency range can also be observed with a specific peak temperature today [16]. Thus the detection of very high frequency thermal gravitational waves is a suitable test to check the possibility of the existence of extra dimensions. Also, the existence of a thermal background of gravitons with a black body type spectrum is discussed in [7-9]. For the gravity-wave background origin, any fit of the CMB anisotropy in terms of gravity background should include a thermal dependence on the spectrum [17]. Based on our work in [18], this thermal spectrum in the low and high frequency ranges causes an enhanced amplitude in the middle range $\sim\left(10^{-16}-10^{9} \mathrm{~Hz}\right)$, which we have called the 'modified amplitude' in [18]. We have analyzed the results of modified amplitude by comparison with the sensitivity of the Adv.LIGO, ET and LISA in [18]. Recently Adv.LIGO has detected the effect of waves of a pair of black holes called GW150914 [19], GW151226 [20] and GW170104 [21]. GW150914 has a peak gravitational-wave strain about $1.0 \times 10^{-21}$ in the frequency range 35 to $250 \mathrm{~Hz}$. There is an average measured sensitivity in the range $\sim\left(10^{-1}-10^{4} \mathrm{~Hz}\right)$ of the Adv.LIGO detectors (Hanford and Livingston) during the time analyzed to determine the significance of GW150914 (Sept 12-Oct 20, 2015) [4]. Therefore in this work, we are interested in upgrading our previous work [18] by comparison of the thermal spectrum with the average measured sensitivity of Adv.LIGO of GW150914 and Dml. We show that there are some chances for detection in the spectrum of RGWs in the usual and thermal cases. 
On the other hand direct detection of the RGWs could, in principle, permit one to directly calculate the inflation field, as has been shown in [22]. Also, different stages of the evolution of the universe (inflation, reheating, radiation, matter and acceleration) cause some variation in the shape of the spectrum of the RGWs. The behaviors of the inflation and reheating stages are often known as power law expansions like $S(\eta) \propto \eta^{1+\beta}, S(\eta) \propto \eta^{1+\beta_{s}}$, respectively. $S$ and $\eta$ are scale factor and conformal time, respectively, and $\beta, \beta_{s}$ are constrained by $1+\beta<0$ and $1+\beta_{s}>0$ [23,24]. The $\beta$ and $\beta_{s}$ have an unique effect on the shape of the spectrum in the full range $\sim\left(10^{-19}-10^{11} \mathrm{~Hz}\right)$ and the high frequency range $\sim\left(10^{9}-10^{11} \mathrm{~Hz}\right)$, respectively. Therefore these two parameters play a main role in the spectrum of the RGWs. Thus, we are interested in obtaining some values on the $\beta$ and $\beta_{s}$ by comparison of the usual and thermal spectra with the average measured sensitivity of the Adv.LIGO and Dml. The obtained values give us more information as regards the evolution of the inflation and reheating stages. In the present work, we use the unit $c=\hbar=k_{B}=1$.

\section{The spectrum of gravitational waves}

The perturbed metric for a homogeneous isotropic flat Friedmann-Robertson-Walker universe can be written as

$\mathrm{d} s^{2}=S^{2}(\eta)\left(\mathrm{d} \eta^{2}-\left(\delta_{i j}+h_{i j}\right) \mathrm{d} x^{i} \mathrm{~d} x^{j}\right)$,

where $\delta_{i j}$ is the Kronecker delta symbol. The $h_{i j}$ are metric perturbations with the transverse-traceless properties, i.e., $\nabla_{i} h^{i j}=0, \delta^{i j} h_{i j}=0$. The gravitational waves are described with the linearized field equation given by

$\nabla_{\mu}\left(\sqrt{-g} \nabla^{\mu} h_{i j}(\mathbf{x}, \eta)\right)=0$.

The tensor perturbations have two independent physical degrees of freedom and are denoted $h^{+}$and $h^{\times}$, which are called polarization modes. We express $h^{+}$and $h^{\times}$in terms of the creation $\left(a^{\dagger}\right)$ and annihilation $(a)$ operators,

$$
\begin{aligned}
h_{i j}(\mathbf{x}, \eta)= & \frac{\sqrt{16 \pi} l_{\mathrm{pl}}}{S(\eta)} \sum_{\mathbf{p}} \int \frac{d^{3} k}{(2 \pi)^{3 / 2}} \epsilon_{i j}^{\mathbf{p}}(\mathbf{k}) \\
& \times \frac{1}{\sqrt{2 k}}\left[a_{\mathbf{k}}^{\mathbf{p}} h_{\mathbf{k}}^{\mathbf{p}}(\eta) e^{i \mathbf{k} \cdot \mathbf{x}}+a_{\mathbf{k}}^{\dagger} h_{\mathbf{k}}^{* \mathbf{p}}(\eta) e^{-i \mathbf{k} \cdot \mathbf{x}}\right],
\end{aligned}
$$

where $\mathbf{k}$ is the comoving wave number, $k=|\mathbf{k}|, l_{\mathrm{pl}}=\sqrt{G}$ is the Planck length and $\mathbf{p}=+, \times$ are polarization modes. The polarization tensors $\epsilon_{i j}^{\mathbf{p}}(\mathbf{k})$ are symmetric and transversetraceless $k^{i} \epsilon_{i j}^{\mathbf{p}}(\mathbf{k})=0, \delta^{i j} \epsilon_{i j}^{\mathbf{p}}(\mathbf{k})=0$ and satisfy the conditions $\epsilon^{i j \mathbf{p}}(\mathbf{k}) \epsilon_{i j}^{\mathbf{p}^{\prime}}(\mathbf{k})=2 \delta_{\mathbf{p} \mathbf{p}^{\prime}}$ and $\epsilon_{i j}^{\mathbf{p}}(-\mathbf{k})=\epsilon_{i j}^{\mathbf{p}}(\mathbf{k})$. Also the creation and annihilation operators satisfy $\left[a_{\mathbf{k}}^{\mathbf{p}}, a_{\mathbf{k}^{\prime}}^{\dagger} \mathbf{p}^{\prime}\right]=$ $\delta_{\mathbf{p} \mathbf{p}^{\prime}} \delta^{3}\left(\mathbf{k}-\mathbf{k}^{\prime}\right)$ and the initial vacuum state is defined as

$a_{\mathbf{k}}^{\mathbf{p}}|0\rangle=0$

for each $\mathbf{k}$ and $\mathbf{p}$. For a fixed wave number $\mathbf{k}$ and a fixed polarization state $\mathbf{p}$ Eq. (2) gives a coupled Klein-Gordon equation as follows:

$f_{k}^{\prime \prime}+\left(k^{2}-\frac{S^{\prime \prime}}{S}\right) f_{k}=0$

where $h_{k}(\eta)=f_{k}(\eta) / S(\eta)[23,24]$ and the prime means a derivative with respect to the conformal time. Since the polarization states are the same, we consider $f_{k}(\eta)$ without the polarization index. The solution of the above equation for the different stages of the universe is given in Appendix A. There is another state called the 'thermal vacuum state'; see Appendix B for more details. The amplitude of the RGWs in the thermal vacuum state is as follows [18]:

$h\left(k, \eta_{0}\right)=A\left(\frac{k}{k_{H}}\right)^{2+\beta} \operatorname{coth}^{1 / 2}\left[\frac{k}{2 T}\right], \quad k \leq k_{E}$,

$h\left(k, \eta_{0}\right)=A\left(\frac{k}{k_{H}}\right)^{\beta-\gamma}\left(1+z_{E}\right)^{\frac{-2-\gamma}{\gamma}} \operatorname{coth}^{1 / 2}\left[\frac{k}{2 T}\right]$,

$k_{E} \leq k \leq k_{H}$,

$h\left(k, \eta_{0}\right)=A\left(\frac{k}{k_{H}}\right)^{\beta}\left(1+z_{E}\right)^{\frac{-2-\gamma}{\gamma}}, \quad k_{H} \leq k \leq k_{2}$,

$h_{m}\left(k, \eta_{0}\right)=A\left(\frac{k_{2}}{k_{H}}\right)^{\beta} \frac{1}{\left(1+z_{E}\right)^{3}}\left(\frac{k}{k_{2}}\right)^{\varrho}, \quad k_{2} \leq k \leq k_{s}$,

with

$$
\begin{aligned}
\varrho & =\frac{\log _{10}\left(h_{2 T}\right)_{k_{s}}-\log _{10}\left(h_{1 T}\right)_{k_{2}}}{\log _{10}\left(k_{s}\right)-\log _{10}\left(k_{2}\right)} \\
& =\frac{\log _{10}\left(\left(\frac{k_{s}}{k_{2}}\right)^{1+\beta} \operatorname{coth}^{1 / 2}\left[\frac{k_{s}}{2 T_{*}}\right]\right)}{\log _{10}\left(\frac{k_{s}}{k_{2}}\right)},
\end{aligned}
$$

where $h_{m}$ is an enhanced amplitude, which is called a modified amplitude in [18] and

$$
\begin{aligned}
h\left(k, \eta_{0}\right)= & A\left(\frac{k}{k_{H}}\right)^{1+\beta-\beta_{s}}\left(\frac{k_{s}}{k_{H}}\right)^{\beta_{s}}\left(\frac{k_{H}}{k_{2}}\right)\left(1+z_{E}\right)^{\frac{-2-\gamma}{\gamma}} \\
& \times \operatorname{coth}^{1 / 2}\left[\frac{k}{2 T_{*}}\right], \quad k_{s} \leq k \leq k_{1} .
\end{aligned}
$$

Here $T=0.001 \mathrm{Mpc}^{-1}$ [7-9], $T_{*}=1.52 \times 10^{25} \mathrm{Mpc}^{-1}$ [16], $\gamma$ is an $\Omega_{\Lambda}$ dependent parameter, and $\Omega_{\Lambda}$ is the energy 
density contrast. We take the value of the redshift $z_{E} \sim 0.3$ and $\gamma \simeq 1.05$ [25] for $\Omega_{\Lambda}=0.692$ from Planck 2015 [2]. By taking the reduced wavelength $\lambda / 2 \pi=1 / H[26,27]$, we can obtain $v_{E} \simeq 3 \times 10^{-19} \mathrm{~Hz}, v_{H} \simeq 3.6 \times 10^{-19} \mathrm{~Hz}$, $\nu_{2} \simeq 1.4 \times 10^{-17} \mathrm{~Hz}, v_{s} \simeq 1.5 \times 10^{9} \mathrm{~Hz}$. The spectral energy density $\Omega_{\mathrm{g}}(v)$ of gravitational waves is defined through the relation $\rho_{\mathrm{g}} / \rho_{\mathrm{c}}=\int \Omega_{\mathrm{g}}(v) \frac{\mathrm{d} v}{v}$, where $\rho_{\mathrm{g}}$ is the energy density of the gravitational waves and $\rho_{\mathrm{c}}$ is the critical energy density and [23]

$\Omega_{\mathrm{g}}(v)=\frac{\pi^{2}}{3} h^{2}\left(k, \eta_{H}\right)\left(\frac{v}{v_{H}}\right)^{2}$.

We can obtain $v_{1} \simeq 4 \times 10^{10} \mathrm{~Hz}$ in order for $\Omega_{\mathrm{g}}\left(v_{1}\right)$ not to exceed the upper bound of the nucleosynthesis rate $\sim 10^{-6}$ [23]. One can get the constant $A$ without scalar running as follows [28]:

$A=\frac{\Delta_{R}\left(k_{0}\right) r^{1 / 2}}{\left(1+z_{E}\right)^{\frac{-2-\gamma}{\gamma}}}\left(\frac{\nu_{H}}{\nu_{0}}\right)^{\beta}$,

where $\Delta_{R}^{2}\left(k_{0}\right)$ is the power spectrum of the curvature perturbation evaluated at the pivot wave number $k_{0}^{p}=k_{0} / a\left(\eta_{0}\right)=$ $0.002 \mathrm{Mpc}^{-1}$ [29] with corresponding physical frequency $v_{0} \simeq 4.9 \times 10^{-19} \mathrm{~Hz} . \Delta_{R}^{2}\left(k_{0}\right)=\left(2.464 \pm 0.072 \times 10^{-9}\right)$ is given by $\mathrm{WMAP} 9+\mathrm{eCMB}+\mathrm{BAO}+H_{0}$ [30]. The tensor to scalar ratio $r<0.11(95 \% \mathrm{CL})$ is based on the Planck measurement [31]. We take $r \simeq 0.1$ and also the value of the redshift $z_{E}=0.3$ for TT, TE, EE + lowP + lensing contribution in this work [2].

\section{The analysis of the spectrum}

Let us now call the spectrum of the waves in the thermal case the 'thermal spectrum'. In this section we have supported and upgraded our previous result obtained in [18] by comparison of the thermal spectrum with the average measured sensitivity of Adv.LIGO (Hanford and Livingstone) and Dml. We are interested in the frequency range $\sim\left(10^{-1}-10^{10} \mathrm{~Hz}\right)$. Therefore we plotted the spectrum by using Eqs. (9)-(11) in Fig. 1. Note that the amplitude is rescaled to $h(v) / \sqrt{v}$ for comparison with Adv.LIGO. Figure 1 shows the spectrum compared to the Hanford (green color) and Livingstone (red color) sensitivity, respectively. The solid and dashed lines stand for the usual and thermal spectrum, respectively. Note that the obtained upper bound of $\beta$ must satisfy the following relation $[23,26,27]$ :

$\left(\frac{\nu_{1}}{v_{H}}\right)^{2+\beta}<\frac{8 \sqrt{\pi}}{A}$

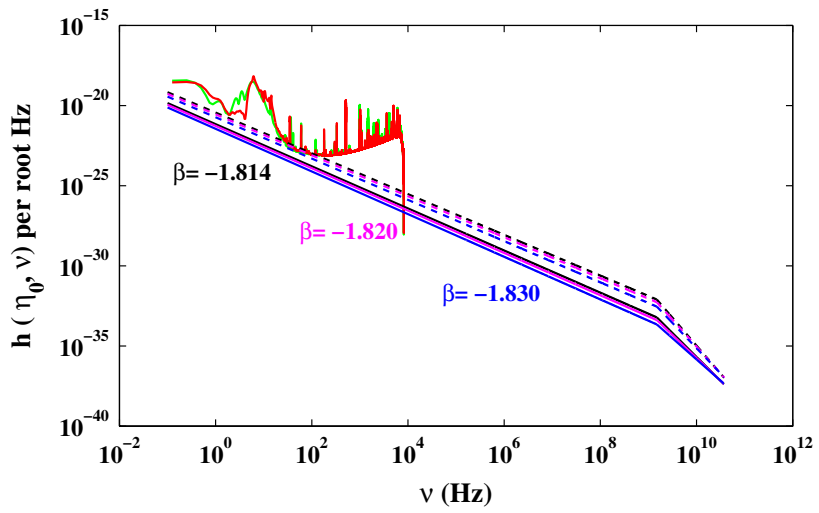

Fig. 1 The spectrum of RGWs in usual case (solid lines) and thermal case (dashed lines) compared to the Hanford (green line) and Livingston (red line) sensitivity

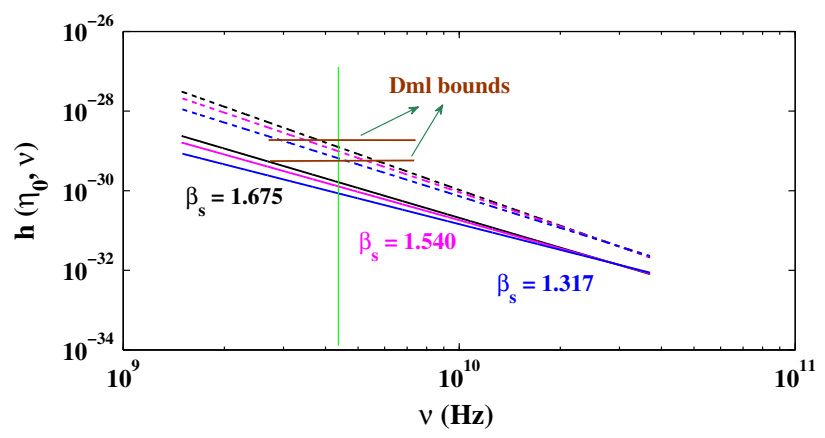

Fig. 2 The high frequency range of Fig. 1 compared to Dml sensitivity at $4.5 \mathrm{GHz}$ (green vertical line)

with $\beta<-1.8140$; see Appendix A for more details. Therefore we plotted the spectrum with some allowed values of $\beta$ in Fig. 1. The obtained diagrams tell us that there are some chances for detection of the thermal spectrum with modified amplitude in addition to the usual spectrum; see the intersection between the dashed lines and Hanford and Livingstone sensitivity in Fig. 1. Also the obtained values of $\beta$ give us more information as regards the evolution of inflation stage; see Eq. (A.2) in Appendix A.

On the other hand there is a procedure for detection based on Dml at GHz band [6]. The author in [6] obtained the minimum sensitivity of the Dml at the frequency $4.5 \mathrm{GHz}$ :

$h_{\min } \sim\left(5.1 \times 10^{-30}-1.6 \times 10^{-29}\right)$.

He believed that the Dml cannot detect the RGWs due to the gap of 4-5 orders between the minimum sensitivity of the Dml and the amplitude of the waves. Therefore he has recommended to upgrade the Dml by using some points that are mentioned in [6]. In addition to those points, we claim that the problem of detection can be removed by considering the thermal spectrum. Using Eq. (11), we plotted thermal spectrum (dashed lines) compared to the usual spectrum (solid 
lines) in the frequency range $\sim\left(10^{9}-10^{10} \mathrm{~Hz}\right)$ in Fig. 2 . The green vertical line stands for the frequency at $4.5 \mathrm{GHz}$. The calculated $\beta_{s}$ in Fig. 2 is obtained corresponding to the values of $\beta$ in Fig. 1 and Eq. (A.14). We can see that the thermal spectrum is within the bound of the Dml at the $4.5 \mathrm{GHz}$. As the author said in [6] the Dml cannot detect the waves in the usual case. But by considering the thermal spectrum, the obtained diagrams tell us that we are lucky to detect the waves at the frequency $4.5 \mathrm{GHz}$. Also the obtained values of $\beta_{s}$ give us more information as regards the evolution of the reheating stage; see Eq. (A.3) in Appendix A.

\section{Discussion and conclusion}

The RGWs are generated before and during the inflation stage. They contain valuable information as regards the early universe in the frequency range $\sim\left(10^{-19}-10^{11} \mathrm{~Hz}\right)$. The thermal spectrum of RGWs causes an enhanced amplitude that is called 'modified amplitude'. The obtained diagrams of the spectrum tell us that there are some chances for detection of the thermal spectrum in addition to the usual spectrum by comparison with the sensitivity of Adv.LIGO of GW150914 and Dml. Also it is observed that $\beta$ and $\beta_{s}$ have an unique effect on the shape of the spectrum. We found some values of $\beta$ and $\beta_{s}$ by using the mentioned comparison with the usual and thermal spectra. Then these values give us more information as regards the evolution of the inflation and reheating stages.

Open Access This article is distributed under the terms of the Creative Commons Attribution 4.0 International License (http://creativecomm ons.org/licenses/by/4.0/), which permits unrestricted use, distribution, and reproduction in any medium, provided you give appropriate credit to the original author(s) and the source, provide a link to the Creative Commons license, and indicate if changes were made.

Funded by SCOAP ${ }^{3}$.

\section{Appendix A}

The general solution of Eq. (5) is a linear combination of the Hankel function with a generic power law for the scale factor $S=\eta^{u}$ given by

$f_{k}(\eta)=A_{k} \sqrt{k \eta} H_{u-\frac{1}{2}}^{(1)}(k \eta)+B_{k} \sqrt{k \eta} H_{u-\frac{1}{2}}^{(2)}(k \eta)$.

We can write an exact solution $f_{k}(\eta)$ by matching its value and derivative at the joining points, for a sequence of successive scale factors with different $u$ for a given model of the expansion of universe. The approximate solution of the spectrum of RGWs is usually computed in two limiting cases based on the waves being outside $\left(k^{2} \gg S^{\prime \prime} / S\right.$, short wave approximation) or inside $\left(k^{2} \ll S^{\prime \prime} / S\right.$, long wave approximation) the barrier. For the RGWs outside the barrier the corresponding amplitude decreases as $h_{k} \propto 1 / S(\eta)$ while for the waves inside the barrier, $h_{k}=C_{k}$ simply is a constant. Therefore these results can be used to obtain the spectrum for the present stage of universe $[23,32]$. The history of the expansion of the universe can be written as follows.

(a) Inflation stage:

$S(\eta)=l_{0}|\eta|^{1+\beta}, \quad-\infty<\eta \leq \eta_{1}$,

where $1+\beta<0, \eta<0$ and $l_{0}$ is a constant.

(b) The $z$-stage:

$S(\eta)=S_{z}\left(\eta-\eta_{p}\right)^{1+\beta_{s}}, \quad \eta_{1}<\eta \leq \eta_{s}$,

where $1+\beta_{s}>0$; see for more details [23]. At the beginning of the reheating stage, the equation of the state of energy in the universe can be quite complicated and is rather model dependent [33]. Hence, this $z$-stage is introduced to allow for a general reheating epoch; see for details [34].

(c) Radiation-dominated stage:

$S(\eta)=S_{e}\left(\eta-\eta_{e}\right), \quad \eta_{s} \leq \eta \leq \eta_{2}$.

(d) Matter-dominated stage:

$S(\eta)=S_{m}\left(\eta-\eta_{m}\right)^{2}, \quad \eta_{2} \leq \eta \leq \eta_{E}$,

where $\eta_{E}$ is the time when the dark energy density $\rho_{\Lambda}$ is equal to the matter energy density $\rho_{m}$. The value of redshift $z_{E}$ at $\eta_{E}$ is $\left(1+z_{E}\right)=S\left(\eta_{0}\right) / S\left(\eta_{E}\right) \sim 1.3$ for TT, TE, EE+lowP+lensing contribution based on Planck 2015 [2] where $\eta_{0}$ is the present time.

(e) Accelerating stage:

$S(\eta)=\ell_{0}\left|\eta-\eta_{a}\right|^{-\gamma}, \quad \eta_{E} \leq \eta \leq \eta_{0}$.

For normalization purpose of $S$, we put $\left|\eta_{0}-\eta_{a}\right|=1$ which fixes the $\eta_{a}$, and the constant $\ell_{0}$ is fixed by the following relation:

$\frac{\gamma}{H_{0}} \equiv\left(\frac{S^{2}}{S^{\prime}}\right)_{\eta_{0}}=\ell_{0}$

where $\ell_{0}$ is the Hubble radius at present with $H_{0}=$ $67.8 \mathrm{~km} \mathrm{~s}^{-1} \mathrm{Mpc}^{-1}$ from Planck 2015 [2]. The physical wavelength is related to the comoving wave number as $\lambda \equiv 2 \pi S(\eta) / k$. If the wave mode crosses the horizon of the universe when $\lambda / 2 \pi=1 / H[26,27]$, then the wave number $k_{H}$ corresponding to the present Hubble radius is $k_{H}=S\left(\eta_{0}\right) / \ell_{0}=\gamma$. There is another wave number, 
$k_{E}=\frac{S\left(\eta_{E}\right)}{1 / H_{0}}=\frac{k_{H}}{1+z_{E}}$, of which the wavelength at the time $\eta_{E}$ is the Hubble radius $1 / H_{0}$. By matching $S$ and $S^{\prime} / S$ at the joint points, one gets

$l_{0}=\ell_{0} b \gamma^{-1} \zeta_{E}^{-\left(1+\frac{1+\beta}{\gamma}\right)} \zeta_{2}^{\frac{\beta-1}{2}} \zeta_{s}^{\beta} \zeta_{1}^{\frac{\beta-\beta_{s}}{1+\beta_{s}}}$

where $b \equiv \gamma^{2+\beta} /|1+\beta|^{(1+\beta)}, \zeta_{E} \equiv \frac{S\left(\eta_{0}\right)}{S\left(\eta_{E}\right)}=\left(\frac{v_{E}}{v_{H}}\right)^{-\gamma}$, $\zeta_{2} \equiv \frac{S\left(\eta_{E}\right)}{S\left(\eta_{2}\right)}=\left(\frac{\nu_{2}}{v_{E}}\right)^{2}, \zeta_{s} \equiv \frac{S\left(\eta_{2}\right)}{S\left(\eta_{s}\right)}=\left(\frac{\nu_{s}}{v_{2}}\right)$, and $\zeta_{1} \equiv \frac{S\left(\eta_{s}\right)}{S\left(\eta_{1}\right)}=$ $\left(\frac{\nu_{1}}{\nu s}\right)^{1+\beta_{s}}$. With these specifications, the functions $S(\eta)$ and $S^{\prime}(\eta) / S(\eta)$ are fully determined $[23,28]$.

The power spectrum of RGWs is defined as

$$
\int_{0}^{\infty} h^{2}(k, \eta) \frac{\mathrm{d} k}{k}=\left\langle 0\left|h^{i j}(\mathbf{x}, \eta) h_{i j}(\mathbf{x}, \eta)\right| 0\right\rangle .
$$

Substituting Eq. (3) in Eq. (A.9) with the same contribution of each polarization, we get

$h(k, \eta)=\frac{4 l_{\mathrm{pl}}}{\sqrt{\pi}} k|h(\eta)|$.

The spectrum at the present time $h\left(k, \eta_{0}\right)$ can be obtained, provided the initial spectrum is specified. The initial amplitude of the spectrum is given by

$h\left(k, \eta_{i}\right)=A\left(\frac{k}{k_{H}}\right)^{2+\beta}$,

where the constant $A$ can be determined by quantum normalization $[23,28]$ as follows:

$A=4 b \frac{l_{\mathrm{pl}}}{l_{0 \sqrt{\pi}}}$.

For the classical treatment of the spacetime during inflation the reduced wavelength $\lambda_{i} /(2 \pi)=1 / H\left(\eta_{i}\right)$ should be larger than the Planck length $\lambda_{i}>l_{\mathrm{pl}}$. Therefore based on Eq. (A.2), the wavelength at the horizon crossing is written as $\lambda_{i}=$ $2 \pi \frac{l_{0}}{b}\left(\frac{v_{H}}{v}\right)^{2+\beta}$. Then we get $[23,26,27]$

$\left(\frac{v}{v_{H}}\right)^{2+\beta}<\frac{8 \sqrt{\pi}}{A}$.

For given $A$ in Eq. (13), we find an upper bound on the $\beta$ at the highest frequency $v_{1}$ as $\beta<-1.8140$. Then we can obtain the allowed values of $\beta_{s}$ by plugging $b / l_{0}$ from Eq. (A.8) into Eq. (A.12) as follows:

$A=\frac{4 l_{\mathrm{pl}}}{\sqrt{\pi}} H_{0}\left(\frac{v_{1}}{v_{s}}\right)^{\beta_{s}-\beta}\left(\frac{v_{s}}{v_{2}}\right)^{-\beta}\left(\frac{\nu_{2}}{v_{E}}\right)^{1-\beta}\left(\frac{v_{E}}{v_{H}}\right)^{-\gamma\left(1+\frac{1+\beta}{\gamma}\right)}$.
Therefore the amplitude of the spectrum for different ranges of wave numbers are given by $[23,24,28]$

$h\left(k, \eta_{0}\right)=A\left(\frac{k}{k_{H}}\right)^{2+\beta}, \quad k \leq k_{E}$,

$h\left(k, \eta_{0}\right)=A\left(\frac{k}{k_{H}}\right)^{\beta-\gamma}\left(1+z_{E}\right)^{\frac{-2-\gamma}{\gamma}}, \quad k_{E} \leq k \leq k_{H}$,

$h\left(k, \eta_{0}\right)=A\left(\frac{k}{k_{H}}\right)^{\beta}\left(1+z_{E}\right)^{\frac{-2-\gamma}{\gamma}}, \quad k_{H} \leq k \leq k_{2}$,

$h\left(k, \eta_{0}\right)=A\left(\frac{k}{k_{H}}\right)^{1+\beta}\left(\frac{k_{H}}{k_{2}}\right)\left(1+z_{E}\right)^{\frac{-2-\gamma}{\gamma}}, \quad k_{2} \leq k \leq k_{s}$,

$\begin{aligned} h\left(k, \eta_{0}\right)= & A\left(\frac{k}{k_{H}}\right)^{1+\beta-\beta_{s}}\left(\frac{k_{s}}{k_{H}}\right)^{\beta_{s}}\left(\frac{k_{H}}{k_{2}}\right)\left(1+z_{E}\right)^{\frac{-2-\gamma}{\gamma}}, \\ & k_{s} \leq k \leq k_{1} .\end{aligned}$

\section{Appendix B}

An effective approach to deal with the thermal vacuum state is the thermo-field dynamics (TFD) [35-38]. In this approach a tilde space is needed besides the usual Hilbert space, and the direct product space is made up of the two spaces. Every operator and state in the Hilbert space has the corresponding counter part in the tilde space [35-38]. Therefore a thermal vacuum state can be defined as

$|T v\rangle=\mathcal{T}\left(\theta_{k}\right)|0 \tilde{0}\rangle$,

where

$\mathcal{T}\left(\theta_{k}\right)=\exp \left[-\theta_{k}\left(a_{\mathbf{k}} \tilde{a}_{\mathbf{k}}-a_{\mathbf{k}}^{\dagger} \tilde{a}_{\mathbf{k}}^{\dagger}\right)\right]$

is the thermal operator and $|0 \tilde{0}\rangle$ is the two mode vacuum state at zero temperature. The quantity $\theta_{k}$ is related to the average number of the thermal particle, $\bar{n}_{k}=\sinh ^{2} \theta_{k}$. The $\bar{n}_{k}$ for given temperature $\mathrm{T}$ is provided by the Bose-Einstein distribution $\bar{n}_{k}=[\exp (k / T)-1]^{-1}$, where $\omega_{k}$ is the resonance frequency of the field. The $a_{\mathbf{k}}, a_{\mathbf{k}}^{\dagger}$ and $\tilde{a}_{\mathbf{k}}, \tilde{a}_{\mathbf{k}}^{\dagger}$, are, respectively, the annihilation and creation operators in Hilbert and tilde space, and they satisfy the usual commutation relations, 
$\left[a_{\mathbf{k}}, a_{\mathbf{k}^{\prime}}^{\dagger}\right]=\left[\tilde{a}_{\mathbf{k}}, \tilde{a}_{\mathbf{k}^{\prime}}^{\dagger}\right]=\delta^{3}\left(\mathbf{k}-\mathbf{k}^{\prime}\right)$. All other commutation relations of these operators are zero. By the appropriate action of the operator (B.21) on $a_{\mathbf{k}}$ and $a_{\mathbf{k}}^{\dagger}$, we obtain [39,40],[41]

$\mathcal{T}^{\dagger} a_{\mathbf{k}} \mathcal{T}=a_{\mathbf{k}} \cosh \theta_{k}+\tilde{a}_{\mathbf{k}}^{\dagger} \sinh \theta_{k}$,

$\mathcal{T}^{\dagger} a_{\mathbf{k}}^{\dagger} \mathcal{T}=a_{\mathbf{k}}^{\dagger} \cosh \theta_{k}+\tilde{a}_{\mathbf{k}} \sinh \theta_{k}$.

Hence the occupation number in thermal vacuum state can be written as follows:

$\left\langle a_{\mathbf{k}}^{\dagger} a_{\mathbf{k}^{\prime}}\right\rangle=\left(\frac{1}{e^{k / T}-1}\right) \delta^{3}\left(\mathbf{k}-\mathbf{k}^{\prime}\right)$.

Thus, using Eq. (3) and Eqs. (B.20)-(B.23) in Eq. (A.9) the power spectrum in the thermal vacuum state is obtained:

$h^{2}(k, \eta)=\frac{16 l_{\mathrm{pl}}^{2}}{\pi} k^{2}|h(\eta)|^{2} \operatorname{coth}\left[\frac{k}{2 T}\right]$.

Therefore in comparison with Eq. (A.11), the spectrum is expressed as

$h\left(k, \eta_{i}\right)=A\left(\frac{k}{k_{H}}\right)^{2+\beta} \operatorname{coth}^{1 / 2}\left[\frac{k}{2 T}\right]$.

The last term becomes significant when the ratio $k /(2 T)$ is less than unity. The wave number $k$ and temperature $T$ are comoving quantities which are related to the physical parameters at the time of inflation; see for details $[7-9,16]$. Thus there is expected an enhancement of the spectrum by a factor $\operatorname{coth}^{1 / 2}[k / 2 T]=\operatorname{coth}^{1 / 2}\left[H S_{i} / 2 T_{i}\right]$, where $S_{i}$ and $T_{i}$ are scale factor and temperature at the beginning of inflation, respectively.

\section{References}

1. C. Corda, Int. J. Mod. Phys. D 18, 2275 (2009)

2. P.A.R. Ade, N. Aghanim et al., Planck Collaboration, arXiv:1502.01589v2 (2015)

3. https://www.elisascience.org/

4. http://losc.ligo.org/events/GW150914/
5. http://www.et-gw.eu

6. M. Tong, Phys. Rev. D 78, 024041 (2008)

7. W. Zhao et al., Phys. Lett. B 680, 411 (2009)

8. K. Bhattacharya et al., Phys. Rev. Lett. 97, 251301 (2004)

9. K. Wang, L. Santos, J.Q. Xia, W. Zhao, Thermal gravitationalwave background in the general pre-inflationary scenario. arXiv:1608.04189 [astro-ph.CO]

10. P. Brax, C. Bruck, Class. Quantum Gravity 20, R201 (2003)

11. T. Kaluza et al., Math. Phys. 1921, 966 (1921)

12. N.A. Hamed, Phys. Lett. B 429, 263 (1998)

13. N. Arkani-Hamed et al., Phys. Rev. D 59, 086004 (1999)

14. L. Randall, R. Sundrum, Phys. Rev. Lett. 83, 3370 (1999)

15. L. Randall, R. Sundrum, Phys. Rev. Lett. 83, 4690 (1999)

16. E.R. Siegel, J.N. Fry, Phys. Lett. B 612, 122 (2005)

17. M. Gasperini, M. Giovannini, G. Veneziano, Squeezed thermal vacuum and the maximum scale for inflation. Phys. Rev. D 48, R439 (1993). arXiv:gr-qc/9306015

18. B. Ghayour, P.K. Suresh, Class. Quantum Gravity 29, 175009 (2012)

19. B.P. Abbott et al., Phys. Rev. Lett. 116, 061102 (2016)

20. B.P. Abbott et al., Phys. Rev. Lett. 116, 241103 (2016)

21. B.P. Abbott et al., Phys. Rev. Lett. 118, 221101 (2017)

22. C. Corda, Gen. Relativ. Gravity 42, 1323 (2010)

23. L.P. Grishchuk, Lect. Notes Phys. 562, 167-194 (2001)

24. Y. Zhang et al., Class. Quantum Gravity 22, 1383-1394 (2005)

25. Y. Zhang et al., Phys. Rev. D 81, 101501 (2010)

26. M. Tong et al., Class. Quantum Gravity 31, 035001 (2014)

27. M. Tong et al., Res. Astron. Astrophys. 16, 49 (2016)

28. M. Tong, Class. Quantum Gravity 29, 155006 (2012)

29. E. Komatsu et al., Astrophys. J. Suppl. 192, 18 (2011)

30. C.L. Bennett, et al., Nine-Year Wilkinson Microwave Anisotropy Probe (WMAP) Observations: Final Maps and Results. arXiv:1212.5225 [astro-ph.CO]

31. Planck Collaboration, Planck 2013 results. XXII. Constraints on inflation. Astron. Astrophys. 571, A22 (2014). arXiv:1303.5082v3

32. Y. Zhang et al., Class. Quantum Gravity 23, 3783-3800 (2006)

33. L.H. Ford, Phys. Rev. D 35, 2955 (1987)

34. L.P. Grishchuk, Class. Quantum Gravity 14, 1445 (1997)

35. H. Umezawa, Y. Yamanaka, Adv. Phys. 37, 531 (1988)

36. H. Fearm, M.J. Collett, J. Mod. Opt. 35, 553 (1988)

37. S. Chaturvedi et al., Phys. Rev. A 41, 3969 (1990)

38. J. Vogt et al., J. Mod. Opt. 38, 2339 (1991)

39. C.T. Lee, Phys. Rev. A 42, 4193 (1990)

40. X.-L. Xu et al., Phys. B 396, 199 (2007)

41. B. Ghayour, P.K. Suresh, Int. J. Mod. Phys. D 22(02), 1350002 (2013) 